\title{
Ubiquitin-dependent regulation of Cdc42 by XIAP
}

\author{
Arun Murali ${ }^{1}$, Jaeyoung Shin ${ }^{1}$, Hajime Yurugi ${ }^{1}$, Aswini Krishnan ${ }^{1}$, Masato Akutsu ${ }^{2}$, Alejandro Carpy $^{3}$, Boris Macek ${ }^{3}$ and \\ Krishnaraj Rajalingam ${ }^{*, 1}$
}

\begin{abstract}
Rho GTPases control fundamental cellular processes and Cdc42 is a well-studied member of the family that controls filopodia formation and cell migration. Although the regulation of $\mathrm{Cdc} 42$ activity by nucleotide binding is well documented, the mechanisms driving its proteostasis are not clear. Here, we demonstrate that the highly conserved, RING domain containing E3 ubiquitin ligase XIAP controls the protein stability of Cdc42. XIAP binds to Cdc42 and directly conjugates poly ubiquitin chains to the Lysine 166 of Cdc42 targeting it for proteasomal degradation. Depletion of XIAP led to an increased protein stability and activity of Cdc42 in normal and tumor cells. Consistently, loss of XIAP enhances filopodia formation in a Cdc42-dependent manner and this phenomenon phenocopies EGF stimulation. Further, XIAP depletion promotes lung colonization of tumor cells in mice in a Cdc42-dependent manner. These observations shed molecular insights into ubiquitin-dependent regulation of Cdc42 and that of actin cytoskeleton.
\end{abstract}

Cell Death and Disease (2017) 8, e2900; doi:10.1038/cddis.2017.305; published online 29 June 2017

Rho GTPases are evolutionarily conserved proteins and make up a unique subfamily within the Ras superfamily of GTPases. They are characterized by the presence of a 'Rho insert domain', which is located between the fifth $\beta$-strand and the fourth $\alpha$-helix of the Rho GTPase. ${ }^{1}$ Like other GTPases, most members of the Rho GTPase family cycle between an inactive GDP bound and an active GTP-bound form. This conversion is catalyzed by guanine nucleotide exchange factors (GEFs), which release bound GDP and facilitates the binding of GTP, and GTPase-activating proteins (GAPs), which inactivate the GTPases by hydrolyzing the GTP back to GDP. Yet another mechanism of inactivating the Rho GTPases is by chaperones called Rho guanine nucleotide dissociation inhibitors (RhoGDIs) that bind to Rho GTPases in the cytosol and sequester them from binding to effectors as well as prevent them from proteolytic degradation. ${ }^{2}$ Rho GTPases can also be regulated by ubiquitination and degradation. ${ }^{3-6}$

Cdc42 is one of the better-studied members of the Rho GTPase family along with Rac1 and RhoA. It has been implicated in a variety of cellular processes including cell motility, cytoskeletal reorganization, filopodia formation, polarity and cell cycle progression. Although the role of Cdc42 in promoting filopodia seems to be cell type specific, several lines of evidence suggest a crucial role for this Rho GTPase in filopodia formation. ${ }^{7,8}$ Further, epidermal growth factor (EGF) has been implicated in Cdc42-dependent protrusion formation. ${ }^{9}$ Here we demonstrate that an evolutionarily conserved member of the inhibitor of apoptosis protein (IAP) family, XIAP functions as a direct E3 ubiquitin ligase of Cdc42 thus controlling its protein stability.

IAPs are a family of multi-functional proteins, primarily characterized by the presence of a baculoviral IAP repeat (BIR) domain, which are classical protein-protein interaction motifs. ${ }^{10}$ Among the eight mammalian IAPs known (X-linked inhibitor of apoptosis protein (XIAP), cellular IAP1/2 (cIAP1 and C-IAP2), melanoma inhibitor of IAP (ML-IAP), Survivin, NAIP, BRUCE and ILP2), five of them also contain a RING finger carboxy terminal domain that confers them with E3 ubiquitin ligase activity. ${ }^{11}$ Ubiquitylation is the most versatile form of post-translational modification wherein Ubiquitin (Ub), a 76 amino acid protein is covalently conjugated to target proteins in a three-step process involving E1, E2 and E3 enzymes. ${ }^{12,13}$ Conjugation of ubiquitin either as a single moiety or as chains of different kinds results in profound effects in cells ranging from protein stability, localization of target proteins to formation of dynamic signaling complexes thus controlling virtually every cellular process. ${ }^{14}$

Apart from the traditional role of IAPs in regulating cell death, recent studies revealed a crucial role of IAPs in controlling inflammation, migration and differentiation. ${ }^{15}$ IAPs (especially XIAP, CIAP1/2 and ML-IAP) were discovered to negatively regulate the stability of CRAF kinase and cell migration. ${ }^{16}$ Further, our recent work has shown that XIAP and cIAP1 can control the plasticity of cell migration by directly regulating the ubiquitination and proteasomal degradation of the Rho GTPase Rac1 ${ }^{4}$ In addition, XIAP can directly conjugate K-63 linked ubiquitin chains to MEKK2 and MEKK3 and interfere with the ERK5 signaling cascade and human myogenic differentiation. ${ }^{17}$ Although the control of $\mathrm{Cdc} 42$ activity by GEFs and GAPs are known and well studied, the molecular mechanisms underpinning the protein degradation of Cdc42 are not clear to date. Here we show that XIAP contributes to $\mathrm{Cdc} 42$ homeostasis by direct ubiquitination leading to protein degradation thus controlling filopodia dynamics and tumor cell metastases. These studies further unveil yet another facet of IAP biology in controlling actin cytoskeleton and tumor metastases.

\footnotetext{
${ }^{1}$ Molecular Signaling Unit-FZI, Institute of immunology, University Medical Center Mainz, JGU-Mainz, Germany; ${ }^{2}$ BMLS, Goethe University, Frankfurt, Germany and ${ }^{3}$ Proteome Center Tuebingen, Interfaculty Institute for Cell Biology, University of Tuebingen, Tuebingen, Germany

*Corresponding author: K Rajalingam, Molecular Signaling Unit-FZI, Institute of immunology, University Medical Center, Langenbeck str 1, Mainz, Rheinland Pfalz 55131, Germany. Tel: +49 613 1178051; Fax: +49 613 1176202; E-mail: Krishna@uni-mainz.de

Received 15.5.17; accepted 23.5.17; Edited by G Melino
} 


\section{Results}

We have previously shown that IAPs, especially XIAP can directly control actin cytoskeleton by regulating Rac1 stability. ${ }^{4}$ We often detected that XIAP-depleted cells exhibited several actin-rich protrusions irrespective of the matrices on which the cells were grown. We thus hypothesized that XIAP might regulate other related Rho GTPases like Cdc42. We first checked whether depletion of XIAP led to any alterations in Cdc42 protein levels. Interestingly, depletion of XIAP led to a striking increase in Cdc42 levels in multiple cell types (Figure 1a). Depletion of XIAP with multiple siRNAs led to a similar increase in Cdc42 levels (Figures $1 \mathrm{~b}$ and c). Further, complementation experiments revealed that expression of XIAP in trans prevented the increase in Cdc42 levels in cells transfected with a siRNA targeted against the 3'UTR of the XIAP mRNA (Figure 1b). Although we consistently detect an increase in the protein levels of Cdc42 upon XIAP knockdown with multiple siRNAs we do not observe a linear correlation between the extent of XIAP depletion and increase in Cdc42 levels. To further corroborate these observations we tested Mouse Embyronic Fibroblasts derived from XIAP-deficient mice. XIAP-deficient MEFs exhibited high Cdc42 protein level, which is reduced after complementation of the same cells with exogenously expressed XIAP cDNA (Figure 1d). Taken together, these results confirm the specificity of the observed phenotype. We performed cycloheximide chase experiments in two different cell lines, HeLa and MDA-MB231 and we detected that loss of XIAP led to increased protein stability of Cdc42 (Figures 1e and f). However, loss of XIAP did not lead to an increase in the mRNA levels of Cdc42 despite strong increase in the protein levels, suggesting that XIAP directly contributes to the proteostasis of Cdc42 (Figure $1 \mathrm{~g}$ ) in multiple cell types.

We often detected that stimulation of either HeLa cells or primary HMEC cells with EGF kinetically led to an increase in the protein levels of Cdc42 (Figures $2 a$ and b). Interestingly, XIAP-depleted cells exhibited high Cdc42 levels despite stimulation with EGF (Figures $2 a$ and $b$ ). We then investigated whether XIAP depletion also affected the activation status of Cdc42 in cells. CRIB pulldown assays employing PAK-PBD in control and XIAP-depleted cells showed that the loss of XIAP also led to an increase in the GTP-bound active form of Cdc42 in normal as well as in tumor cells (Figures $2 \mathrm{c}$ and $\mathrm{d}$ ). Intriguingly, we also detected XIAP co-precipitating with active Cdc42 suggesting that XIAP and Cdc42 co-exist in a protein complex (Figures $2 \mathrm{c}$ and $\mathrm{d}$ ). We then tested for phenotypic changes in XIAP-depleted cells as Cdc42 has been well documented to control filopodia formation in multiple cell types.

As expected, XIAP depletion led to profuse filopodia formation in both primary and immortalized HMEC cells (Figures $3 a$ and b). We also observed the same phenomenon in other tumor cell lines such as $\mathrm{NCl}-\mathrm{H} 226$ (Figure $3 \mathrm{~b}$ and Supplementary Figure 1A). Further, co-depletion of Cdc42 reduced the number of filopodia in both control and XIAPdepleted cells confirming the specificity of this phenotype (Figure 3c and Supplementary Figures 1B-1D). We detected that XIAP-depleted cells phenocopied EGF stimulated cells with an increased Cdc42 activity (Figures $3 d$ and e).
Immunocytochemical studies employing a validated Cdc42 antibody revealed that Cdc42 is strongly upregulated in XIAPdepleted cells and localizes to the filopodial protrusions in immortalized HMEC cells (Figure $3 d$ ). These results confirmed that loss of XIAP led to an increase in the total as well as active levels of Cdc42 thus contributing to the enhanced actin-rich protrusions in these cells.

We then investigated the molecular mechanisms behind XIAP-mediated Cdc42 degradation. Interestingly, we detected that XIAP directly binds to Cdc42 in a RING domaindependent manner (Supplementary Figure 2A). XIAP failed to interact with the Cdc42T17N mutant in vitro, suggesting that the observed interaction may be dependent on the activation status of Cdc42 (Figure 4a). We then explored the possibility whether XIAP is a direct E3 ubiquitin ligase of Cdc42. In vitro ubiquitination experiments revealed that XIAP could directly conjugate ubiquitin to wild-type and CdcQ61L mutants efficiently (Supplementary Figure 2B), consistent with the interaction experiments. Further experiments also revealed that XIAP but not cIAP1 could directly conjugate poly ubiquitin chains to the active Cdc42Q61L mutant (Figure 4b). We then tested if XIAP can influence the ubiquitination and proteasomal degradation of Cdc42 at endogenous levels. To pursue these studies, we performed pulldown experiments including TUBEs (tandem ubiquitin binding entity) to enrich ubiquitinated proteins from control and XIAP-depleted cell lysates as mentioned in the methods section. Interestingly, we detected that loss of XIAP reduced the polyubiquitination of Cdc42 at endogenous levels (Figure 4c). Previous studies with bacterial toxins have suggested that activated Rho GTPases are often targeted for proteosomal degradation. ${ }^{18} \mathrm{We}$ then tested if persistent activation of Cdc42 led to its decreased protein stability. Consistent with the observations made with Rac1, cycloheximide chase experiments revealed that the activated Q61L form of Cdc42 was less stable when compared to either wild-type Cdc42 or the Cdc42T17N inactive mutants (Supplementary Figure 2C).

We then employed mass spectrometric analysis to identify the possible ubiquitination sites of Cdc42. These analyses revealed that XIAP conjugated ubiquitin chains to the K166 of Cdc42 which is localized to the C-terminus of the Rho GTPase (Figure 4d). Consistently, mutation of K166 (Cdc42Q6 $1 \mathrm{LK} 166 \mathrm{~A})$ significantly enhanced the protein stability of Cdc42Q61L suggesting that ubiquitination at this site by XIAP is relevant for the proteolysis of activated Cdc42 (Supplementary Figure 2D).

As XIAP can directly ubiquitinate Cdc42, we tested if the RING domain of XIAP is required for modulating the protein levels of Cdc42 in vivo. To perform these studies, we employed MEFs derived from XIAP-deficient mice and complimented them with either wild-type or RING (XIAPH467A) mutants. Interestingly, expression of wild-type XIAP but not the RING mutant of XIAP reduced the Cdc42 levels in XIAP-deficient MEFs (Figure 4e). In fact, expression of the RING mutant of XIAP augmented the protein levels of Cdc42 under these settings (Figure 4e). These results further confirm that XIAP directly influences the protein stability of Cdc42 through its E3 ligase activity.

We performed molecular modeling studies based on the published crystal structures of Cdc42 and PAK6, which 
a

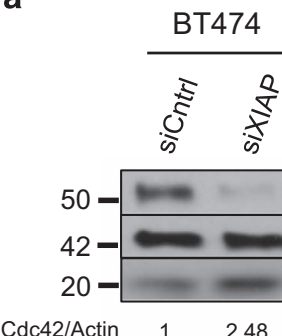

$\frac{\text { HeLa }}{\text { 妾 }}$

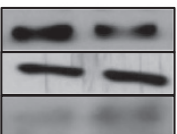

$\mathrm{NCl}-\mathrm{H} 226$ MDA-MB-231
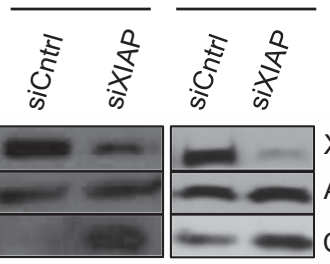

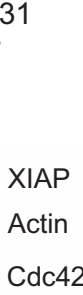

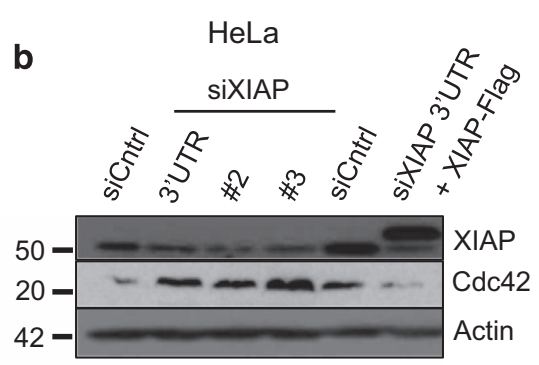

C

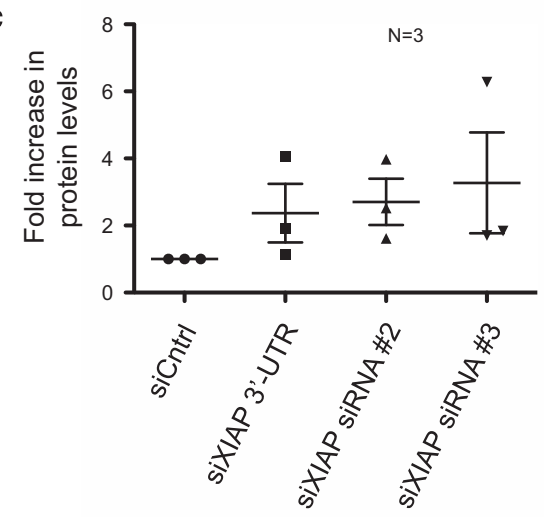

d



e
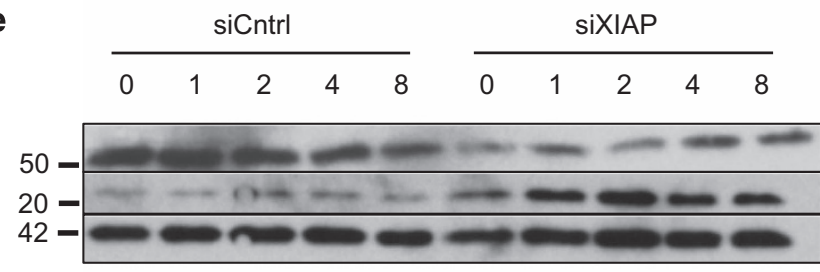

f

MDA-MB231

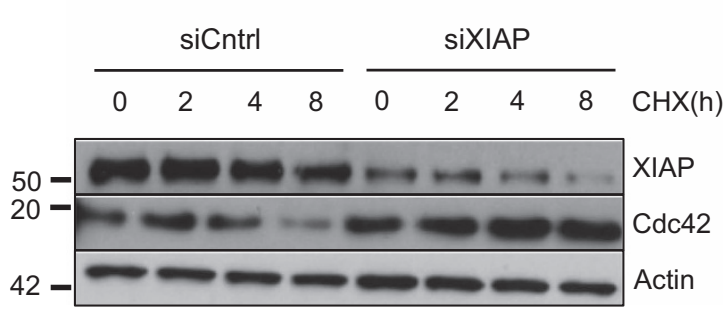

$\mathrm{CHX}(\mathrm{h})$

XIAP

Cdc42

Actin

Figure 1 Depletion of IAPs leads to up-regulation of Cdc42 (a) BT474, HeLa, NCI-H226 and MDA-MB231 cells were transfected with Control or XIAP siRNA for 48 h and lysates were then collected for immunoblotting. The levels of total Cdc42 were monitored in the immunoblots. Quantification was performed by densitometry (as described in Materials and Methods). (b) HeLa cells were transfected with three different XIAP siRNAs and Cdc42 levels were monitored. Further, HeLa cells transfected with the 3'-UTR XIAP siRNA were complemented with the XIAP-Flag vector and Cdc42 levels were observed. (c) Quantification of three independent experiments where three different XIAP siRNAs were used and Cdc42 protein expression was checked $48 \mathrm{~h}$ post transfection (d) Mouse embryonic fibroblasts (MEFs) were cultured and lysed to check for Cdc42 levels. Control MEFs and XIAP knockout MEFs stably complemented with Flag tagged XIAP were employed. (e, f) Cycloheximide chases were performed in HeLa (e) and MDA-MB231 (f), and the stabilization of Cdc42 upon depletion of XIAP was observed. (g) qPCR performed with the three different XIAP siRNAs to observe the transcriptional regulation of Cdc42. Data shown are from three independent experiments

led to the prediction that the conjugation of Ubiquitin to K166 could disrupt the interaction between Cdc42 and its effector protein PAK (Figure 5a). Consistently, CRIB pulldown experiments involving the p21-binding domain (PBD) of PAK revealed that ubiquitinated $\mathrm{Cdc} 42$ failed to interact with PAKPBD. Cdc42 was first subjected to an in vitro ubiquitination assay and GST PAK-PBD was subsequently added to the reaction mix and the CRIB pulldown was performed as 
a

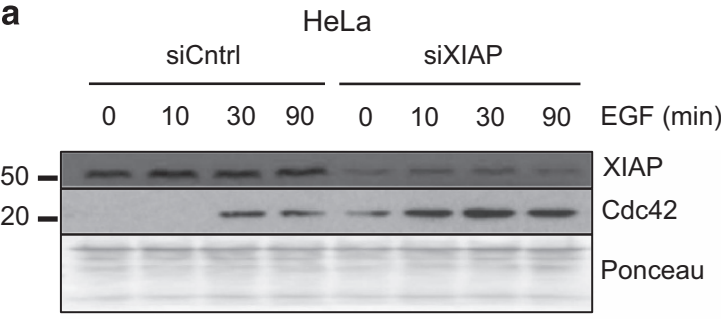

C


b

HMEC

siCntrl siXIAP

0 10'30' $1 \mathrm{~h} 2 \mathrm{~h} 4 \mathrm{~h} 8 \mathrm{~h} 0$ 10' $30^{\prime} 1 \mathrm{~h} 2 \mathrm{~h} 4 \mathrm{~h} 8 \mathrm{~h}$ EGF (min)

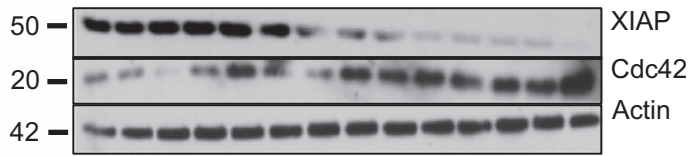

d
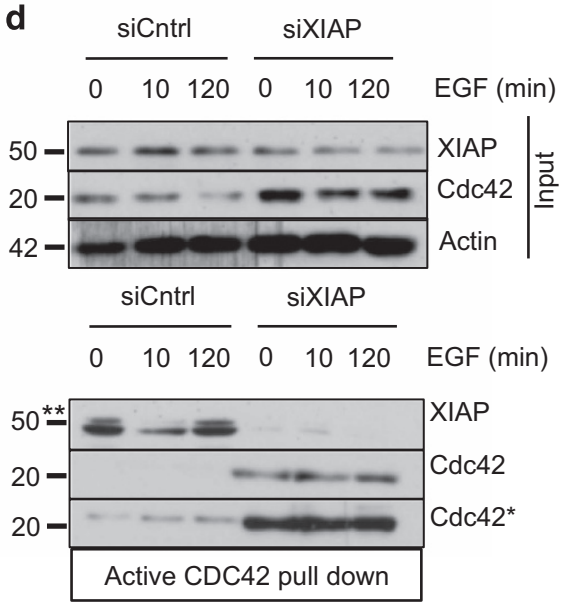

Figure 2 EGF treatment further enhances the stability of Cdc42 upon XIAP depletion (a) HeLa cells and (b) HMECs were transfected with XIAP siRNA and treated with EGF $48 \mathrm{~h}$ post transfection for the times indicated in the figure. The levels of $\mathrm{Cdc} 42$ were monitored via immunoblotting. (c, d) Active Cdc42 pulldown using GST PAK-PBD protein was performed in HeLa and HMECs to test for levels of the active protein upon EGF treatment at times indicated in the figure. * denotes a higher exposure of Cdc42 in (d). ${ }^{*}$ denotes the upper non-specific band in the XIAP blot

described in the methods. We observed that ubiquitinated Cdc42 failed to interact with PAK-PBD, suggesting that conjugation of ubiquitin to K166 directly prevented the interaction of $\mathrm{Cdc} 42$ with its downstream effector (Figure 5b).

Previous studies have shown that Cdc42 has a crucial role in the metastases of tumor cells. ${ }^{19}$ To evaluate the pathophysiological significance of XIAP-Cdc42 interaction in vivo, we employed an experimental metastases model employing NOD/SCID mice. Control or XIAP-depleted HeLa cells were injected into the tail vein of mice, and we detected that there was an increase in the number of nodules in the surface of the lung at 4-5 weeks post injection in mice injected with XIAP-depleted cells (Figure 6a). Co-depletion of Cdc42 strongly reduced the number of lung nodules, confirming that loss of XIAP led to an increase in the number of lung nodules in a Cdc42-dependent manner (Figure 6b). Hemotoxylin and Eosin staining confirmed the presence of nodules in the lungs of the mice (Figure 6c). These results reveal the first E3 ubiquitin ligase of Cdc42 and shed further insights into the molecular mechanism behind XIAP-dependent regulation of actin-rich protrusions and tumor cell invasion.

\section{Discussion}

Rho GTPases are crucial regulators of cell adhesion and migration and the spatiotemporal dynamics of activation has been intensively studied. GEFs and GAPs regulate the activation cycle of Rho GTPases and ubiquitin-dependent inactivation has recently emerged as a 'non-canonical' means of Rho GTPase inactivation. RhoA has been shown to be ubiquitinated by Smurf1 and FBXL19 while HACE1, FBXL19 and XIAP/CIAP1 were shown to be the E3 ubiquitin ligases of Rac1 targeting these Rho GTPases for proteasomal degradation., ${ }^{4,20-22}$ Rac1 is also SUMOylated by PIAS and this is an essential event for optimal cell migration in response to HGF. ${ }^{23}$ Cdc42 was discovered nearly 25 years ago and its role in regulation of actin cytoskeleton, polarity, cell adhesion and migration is well documented. Cdc42 has been best characterized for its ability to stimulate filopodia formation at the leading edge of the migrating cells. Like other Rho GTPases, binding to RhoGDls also regulates Cdc42 activity and protein stability. As the activated form of Cdc42 is targeted for ubiquitination and proteasomal degradation, it is possible that interaction with XIAP and ubiquitination is also regulated by interaction with RhoGDI. Further, the RING domain of XIAP has also been shown to interact with RhoGDI. ${ }^{24}$ As XIAP conjugates ubiquitin to the C-terminus, one could envisage that ubiquitinated species of Cdc42 may not interact with RhoGDI. Depletion of XIAP also enhanced the active GTP-bound form of Cdc42 with a concomitant increase in actin-rich filopodial protrusions in normal and tumor cells. As XIAP binds to the activated Cdc42 and promotes ubiquitination, loss of XIAP led to the accumulation of activated Cdc42 in cells. These observations further support the model that XIAP-mediated ubiquitination 
a



b

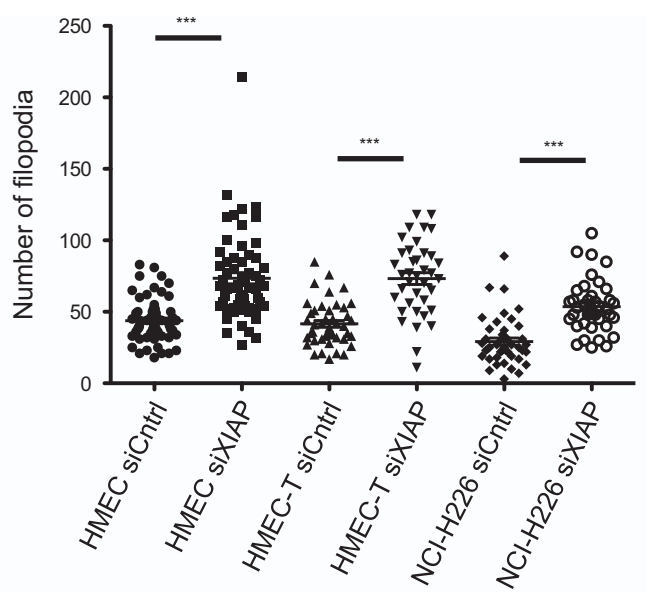

d
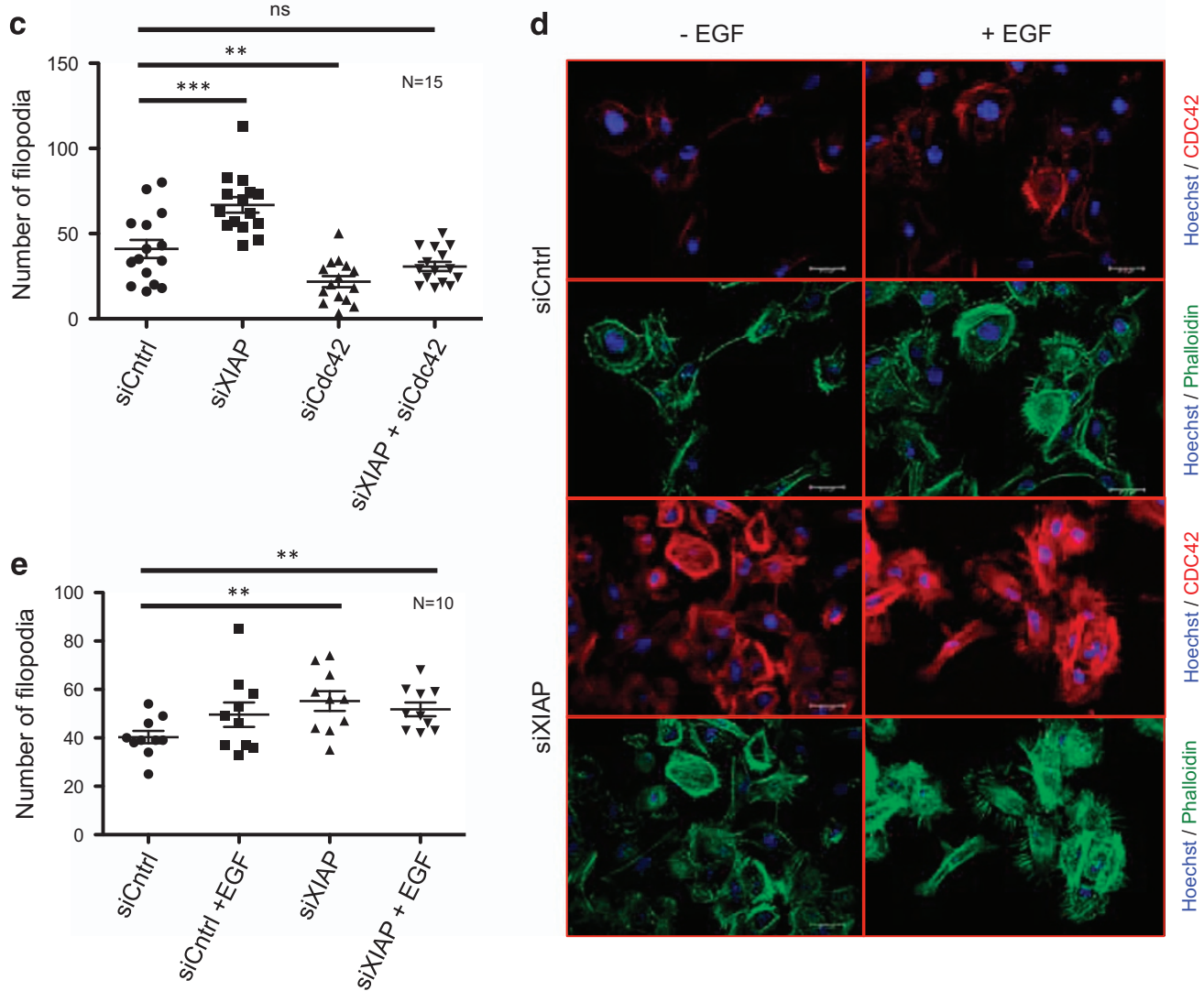

Figure 3 Depletion of XIAP increases the number of Filopodia (a) HMEC, NCI-H226 and HMEC-T cells were depleted of XIAP and immunofluorescence staining was performed using Phalloidin AlexFluor 488 to look for differences in actin structures. (b) Quantification of the number of filopodia in the three cell lines of (a) upon depletion of XIAP. Data shown are from three independent experiments with a total of 40-60 cells counted per condition (see Materials and Methods for details), $P<0.001$ (c) HMEC-T cells were depleted of XIAP as well as Cdc42, the quantification of the number of filopodia is shown, $n=15$ cells. (d) HMEC-T cells were treated with EGF stimulation after Control or XIAP siRNA treatment and immunofluorescence was performed using three different stains: Phalloidin for Actin, Hoechst for the nucleus and a Cdc42 primary antibody followed by Cy3 mouse secondary to check for localization of Cdc42 protein in the cell (e) Quantification of the number of filopodia seen in Figure (d) with $n=10$ cells counted per condition, $P<0.01$

could serve as a non-canonical way of Cdc42 inactivation. Further studies are clearly warranted to decipher the spatiotemporal dynamics of XIAP-Cdc42 interaction. We observe that XIAP directly interacts with Cdc42 and co-precipitated with the activated form of $\mathrm{Cdc} 42$. It would be interesting to test if any other effectors of Cdc42 trigger the RING activity of XIAP.
XIAP is a legitimate caspase inhibitor and thus efforts have been made to target XIAP-caspase inhibition to promote tumor cell apoptosis. Several drugs have been developed to achieve these goals and some of them have reached the clinics. Apart from XIAP, cIAP1 has also been shown to regulate Rho GTPases. ClAP1 can function as an E3 ubiquitin ligase of Rac1 and control Cdc42 activity in response to TNF alpha. ${ }^{4,25}$ 
His-TUBE Ni-NTA pull down

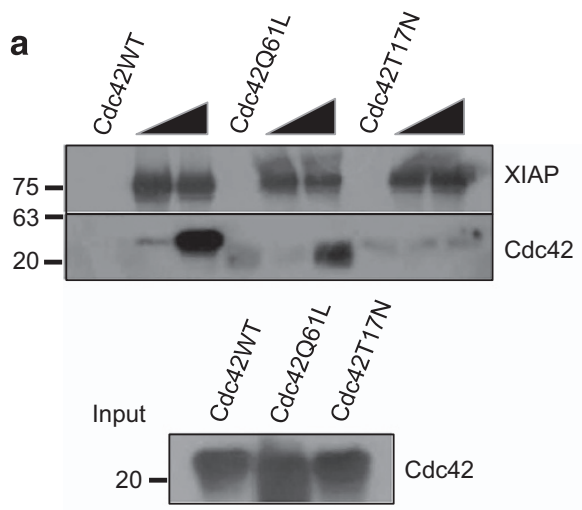

c +MG132

Total cell lysates +MG132

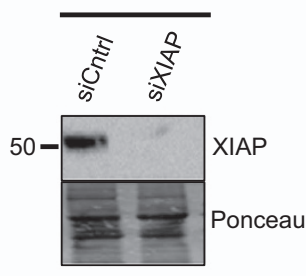

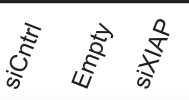

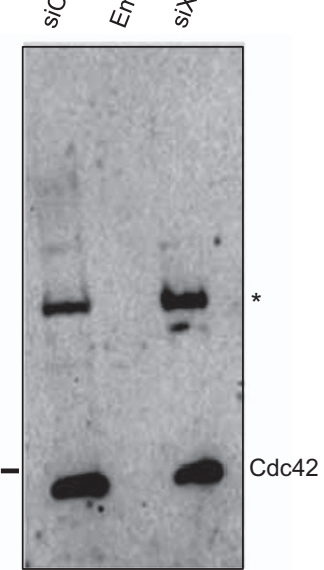

b

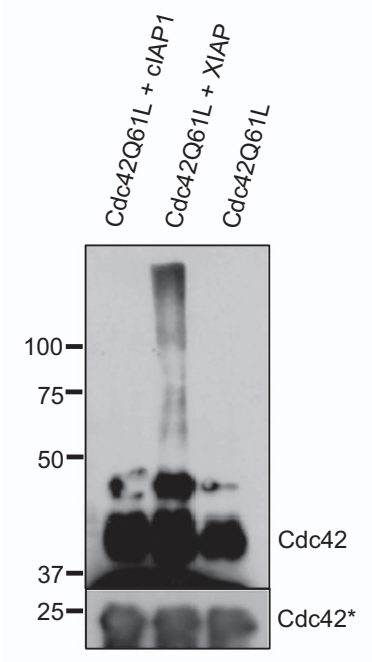

d

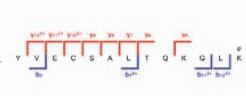

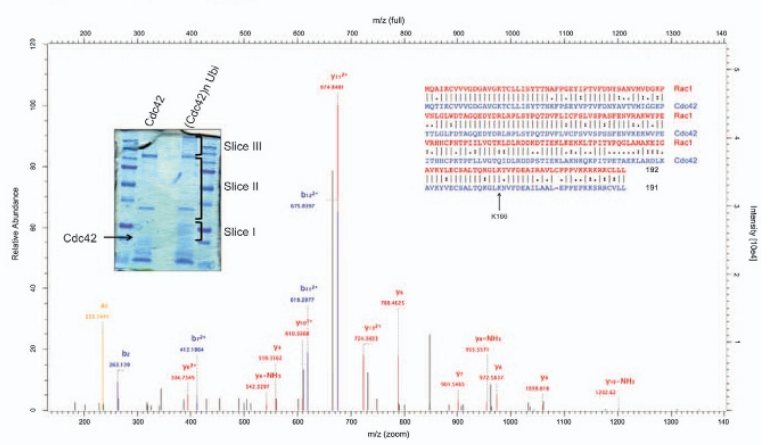

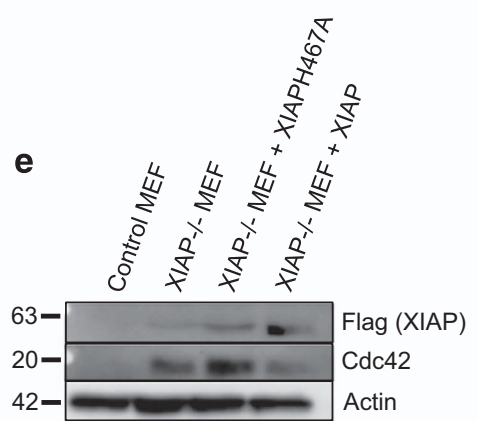

Figure 4 (a) Interaction between XIAP and different Cdc42 mutants was tested via an in vitro GST Pulldown assay. With GST protein as a control, two different concentrations of cleaved Cdc42 mutants were used to test the binding to GST tagged XIAP (b) In vitro ubiquitination of Cdc42 by XIAP. Purified recombinant Cdc42Q61L was subjected to in vitro ubiquitination by XIAP and cIAP1 recombinant proteins (protocol described in Materials and Methods). (c) HeLa cells were transfected with XIAP siRNA for $48 \mathrm{~h}$ and treated with MG132 for $6 \mathrm{~h}$. The cells were lysed in RIPA buffer and His-TUBE immobilized on Ni-NTA beads were employed to enrich the ubiquitinated proteins. The samples were loaded onto a gel and the presence of Cdc42 was monitored by immunoblots. The efficiency of XIAP knockdown was tested in the lysates control. * denotes an unspecific band. (d) Gel slices of the in vitro ubiquitination reaction were subjected to mass spectrometric analysis to determine the Lysine(s) responsible for the ubiquitination. Inset shows the gel slices taken for the analysis as well as a comparison between the sequences of Rac1 and Cdc42 (e) Mouse embryonic fibroblasts (MEFs) were cultured and lysed to check for Cdc42 levels. Control MEFs and XIAP knockout MEFs stably complemented with different XIAP constructs were used for this experiment. * denotes lower exposure of Cdc42

Figure 5 (a) Ribbon model of crystal structure of CDC42 (Yellow) in complex with PAK6 (Purple) (PDB: 2ODB). P-loop (residues 10-17), Switch I (residues 32-40), and switch II (residues 60-67) of Cdc42 are green, red and cyan, respectively. Nucleotide (cyan), K166 of CDC42 and residues 11-16 of PAK6 are shown in ball-and-stick representation. Hydrogen bond between K166 of CDC42 and PAK6 (carbonyl oxygen of I12) is shown as black dashed line. The second figure shows a model of ubiquitinated (Green) CDC42 (Yellow) in complex with PAK6 (Purple). Finally, a sequence alignment of CRIB domain of PAK2 and PAK6. (b) The same in vitro ubiquitination assay as in Figure 4b was performed and the reaction was then subjected to a PAK-PBD GST pulldown (as described in Materials and Methods) to check for interaction of ubiquitinated Cdc42 and its downstream effector PAK. The ubiquitination of Cdc42 was confirmed in the samples employed for PAK-PBD pulldown (lane 4) 


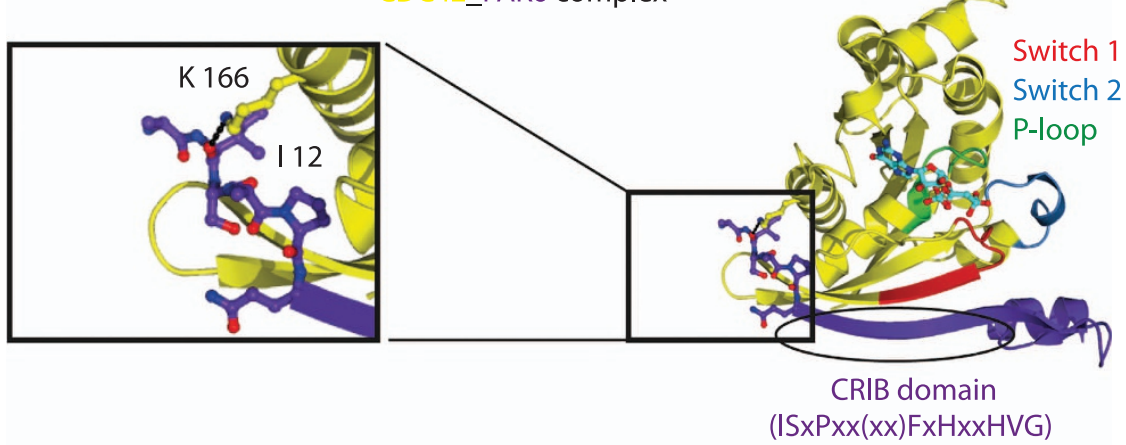

PAK6 complex

K166 ubiquitination model
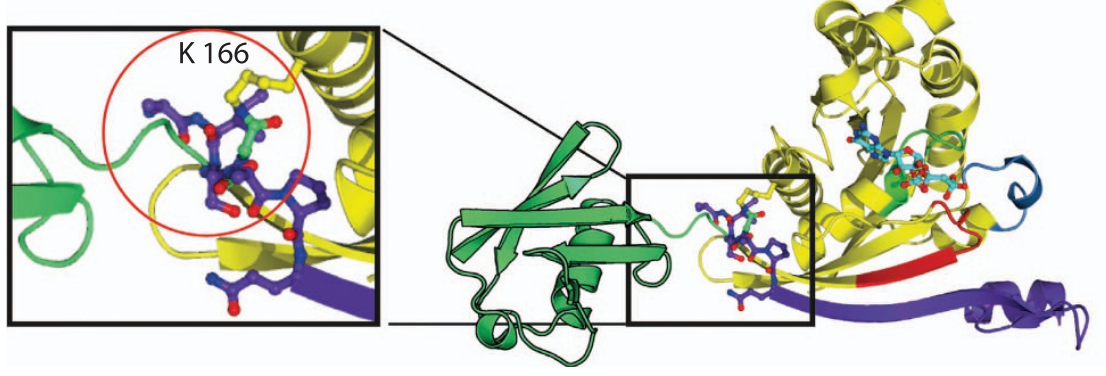



\footnotetext{
CRIB domain

(ISxPxx(xx)FxHxxHVG)
}




a

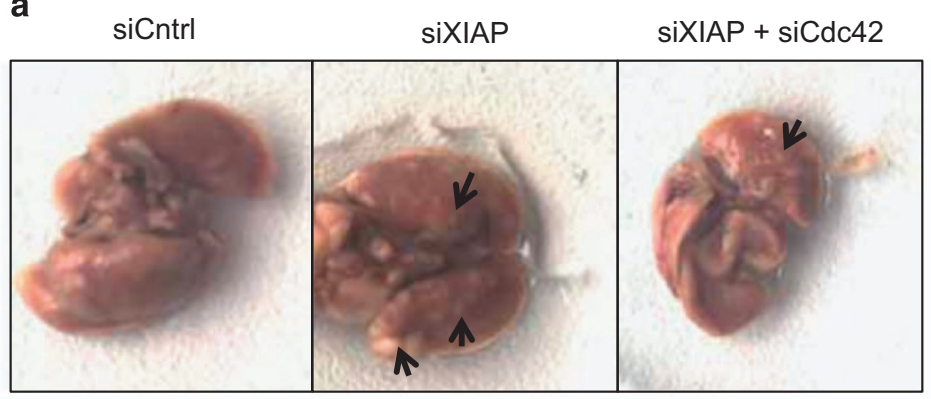

C

C Lung - 1

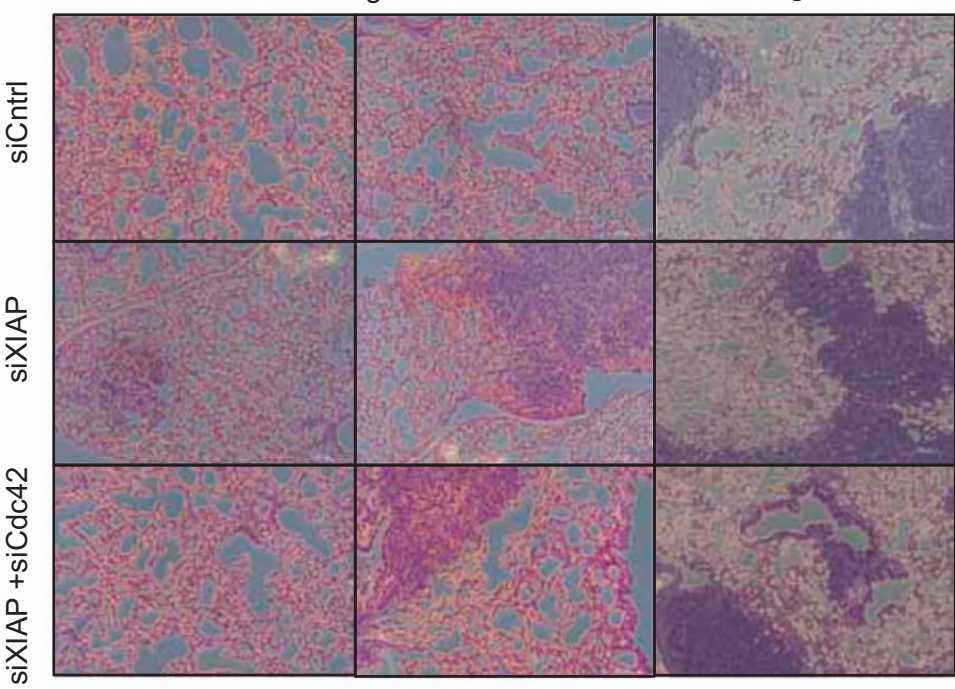

b

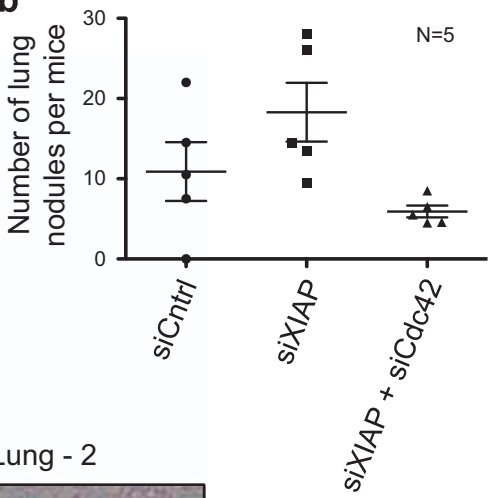

d


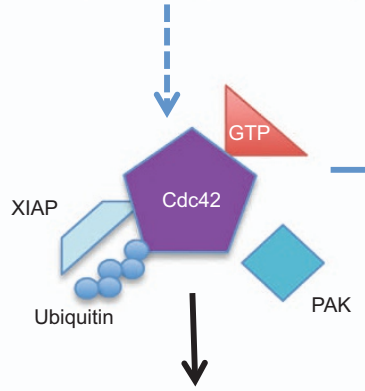

Degradation



Enhanced stability

Figure 6 XIAP depletion in tumor cells promotes lung colonization of mice in a Cdc42-dependent manner (a) NOD-SCID mice were injected through the tail vein with 1 million tumor cells treated with different siRNAs as indicated. Mice were killed after 4-5 weeks of injection and the lungs were isolated. Shown are pictures of the whole lung (b) Metastatic nodules were counted manually on each lung by three independent pairs of eyes, with five mice lungs counted per condition (c) Lungs were fixed and $\mathrm{H} \& \mathrm{E}-$ stained and representative images of the lungs are shown. (d) Proposed model for the interaction between XIAP and Cdc42

However, we found that loss of clAP1 did not lead to an increase in the levels of Cdc42. Consistently, we failed to detect any potential ubiquitination of Cdc42 by clAP1 (Figure 4b). Currently it is unclear about the kind of ubiquitin chains synthesized by XIAP on Cdc42. However, our experiments with TUBEs reveal that polyubiquitination of $\mathrm{Cdc} 42$ is dependent on the
RING activity of XIAP in vivo at endogenous levels. In conclusion, our studies revealed the other side of XIAP in the control of Rho GTPases (especially Rac1 and Cdc42), the prime drivers of tumor cell migration and invasion. Regulation of Cdc42 via GEFs and GAPs is well understood; our study identifies XIAP as the first E3 ubiquitin ligase of Cdc42 and adds 
another dimension to the inactivation of this Rho GTPase and ubiquitin-dependent regulation of actin cytoskeleton.

\section{Materials and Methods}

Transfection of siRNAs and plasmids. To silence XIAP, CIAP1 and Cdc42 expression by RNA interference, $~ 75000$ cells/well were seeded in a 12well plate at least $20 \mathrm{~h}$ prior to transfection. siRNAs directed against various genes and scrambled control siRNA as negative control were transfected using Hiperfect (Qiagen, Hilden, Germany) or Saint Red (Synvolux, Leiden, Netherlands) or Lipofectamine RNAimax (Invitrogen, Schwerte, Germany) transfection kits. The transfection was performed according to manufacturer's instructions. At $24 \mathrm{~h}$ post transfection, cells were trypsinized and one half of the cells were seeded out on glass coverslips in a 12-well plate for immunofluorescence analysis if needed, while the other half was seeded for western blot analysis. The cells were normally lysed $48 \mathrm{~h}$ post transfection. Unless otherwise mentioned, siRNAs have been transfected at $60 \mathrm{nM}$. The following siRNAs were employed in this study: XIAP3'-UTR siRNA: Target Sequence: CTGACTGATCTAATTGTATTA (Qiagen), XIAP siRNA-2: Target Sequence: GAAGGAGAUACCGUGCGGUGCUUUA (Invitrogen), XIAP siRNA-3: Target Sequence: AAGTGCTTTCACTGTGGAGGA (Qiagen), Control siRNA: Target Sequence: AATTCTCCGAACGTGTCACGT(Qiagen), Cdc42 siRNA-1: Target Sequence: CATCAGATTTGAAATATTTAA (Qiagen), Cdc42 siRNA-2: Target Sequence: GGCGATGGTGCTGTTGGTAAA (Qiagen).

In the case of overexpression or complementation, plasmids at a stock concentration of $1 \mu \mathrm{g} / \mathrm{ml}$ were taken and transfected into cells using PEI transfection reagent. A day after transfection was sufficient to observe complemented proteins in the tumor cells. In the case of Cycloheximide chases post overexpression, the chase experiments were started $40 \mathrm{~h}$ post transfection so as to lyse cells $48 \mathrm{~h}$ post transfection. For stable shRNA-mediated knockdown Lentiviral particles (mission shRNA) carrying control (Sigma, Taufkirchen, Germany) and Cdc42 shRNA, Sequence: CCGGCCTGATAT CCTACACAACAAACTCGAGTTTGTTGTGTAGGATATCAGGTTITG, were obtained from Sigma and the stable cell lines were produced following the manufacturer's instructions. In case of co-knockdown experiments, cells were transfected with siRNAs at a final concentration of $60 \mathrm{nM}$ each.

Rho GTPase activation assays. XIAP was knocked down in HeLa and HMEC cells and $48 \mathrm{~h}$ post transfection, cells were lysed. EGF treatments were performed at the times indicated prior to lysis of cells. The GTP-bound Cdc42 was precipitated using the Cdc42 Active PD kit from Thermo Fisher Scientific, Darmstadt, Germany. This was done according to manufacturer's instructions.

Ubiquitylation assays. In vitro ubiquitylation of Cdc42 was performed with Cdc42 Wild Type, Cdc42Q61L or Cdc42T17N purified from bacteria. The ubiquitylation reaction was performed in the presence of Ubiquitylation buffer (50 mM Tris- $\mathrm{HCl}, \mathrm{pH} 7.5,100 \mathrm{mM} \mathrm{NaCl}, 2.5 \mathrm{mM} \mathrm{MgCl}$, 1 mM DTT), $100 \mathrm{nM}$ XIAP (R\&D Systems, Minneapolis, MN, USA), $100 \mathrm{nM}$ E1 (Boston Biochem, Cambridge, MA, USA), $150 \mathrm{mM}$ UbcH5a (Boston Biochem), $107 \mathrm{mM}$ His-Ubiquitin (Boston Biochem), 50 mM EDTA, 1× Mg-ATP (Enzo Life Sciences, Lörrach, Germany), $1 \mathrm{U}$ inorganic pyrophosphatase (Fluka, Taufkirchen, Germany) and $50 \mathrm{mM}$ DTT. The reaction was incubated at $37^{\circ} \mathrm{C}$ for $30 \mathrm{~min}$. For western blot analyses, the reaction was stopped using Laemmli buffer and Cdc42 was visualized using Cdc42 antibody (BD, Heidelberg, Germany).

Immunofluorescence and counting of filopodia. Cells were seeded and transfected in 12-well plates. One day post transfection, they were split and re-seeded upon coverslips in a new 12-well plate. The confluency of cells on the cover slip was maintained below $30 \%$. Two days post transfection, cells were taken and washed thrice with PBS before being fixed in $4 \%$ paraformaldehyde for $10 \mathrm{~min}$. They were then permeabilised using $0.1 \%$ Triton $\mathrm{X}-100$ for $3 \mathrm{~min}$ and then blocked for 20 min at RT using $1 \%$ BSA in PBS. The actin cytoskeleton was then labeled with 1:50 Alexa Fluor 488 Phalloidin (Invitrogen) in blocking buffer for 20 min in the dark. For staining of $\mathrm{Cdc} 42$ protein, cells were incubated in 1:250 dilution of $\mathrm{Cdc} 42$ antibody (BD) overnight in $0.5 \%$ BSA in PBS. Cells were then incubated with secondary Cy3 antibody in Blocking Buffer for $1 \mathrm{~h}$ at RT in the dark. Between each step, the cover slip was always washed thrice with PBS. Finally, the cells were mounted on glass slides using Moviol (Sigma) and examined using a Confocal microscope (Leica (Wetzlar, Germany) TCS SP8). Cells were normally viewed at $\times 63$ magnification and digital zooming was done in case single cells were focused on. Filopodia were then counted manually using the KatiKati software, which has an in-built zoom function to enlarge the images. This was done for all the cell types, tumor and primary cell lines employed in the paper.

Cell culture. HeLa, BT474 and MDA-MB231 cells were cultured in DMEM medium (Gibco, Schwerte, Germany) supplemented with 10\% FCS (Gibco) and $0.2 \%$ Penicillin $(100 \mathrm{U} / \mathrm{ml}) /$ streptomycin $(100 \mu \mathrm{g} / \mathrm{ml})(\mathrm{Gibco})$ at $37^{\circ} \mathrm{C}$ in $5 \% \mathrm{CO}_{2}$. $\mathrm{NCl}-\mathrm{H} 226$ cells were cultured in RPMI (Gibco) medium supplemented with $10 \%$ FCS and antibiotics, while HMEC and HMEC-T cells were cultured in MEGM medium (Lonza, Cologne, Germany) supplemented with growth factors as recommended by the company. For treatment with EGF (Labgen, Germany), a concentration of $25 \mathrm{pg} / \mu$ l was used. MG132 treatment (Calbiochem, Darmstadt, Germany) was performed $5 \mathrm{~h}$ before lysing of cells at a concentration of $10 \mu \mathrm{M}$. Cycloheximide chases were performed with InSolution Cycloheximide (Calbiochem) at a final concentration of $100 \mu \mathrm{g} / \mathrm{ml}$.

Western blot analysis. Cells were lysed in $5 \times$ Laemmli Buffer and boiled at $100{ }^{\circ} \mathrm{C}$ for $5 \mathrm{~min}$ before loading them on the polyacrylamide gels. After separation by SDS-PAGE, the proteins were transferred to either nitrocellulose or PVDF membranes (GE Healthcare Life Sciences, Freiburg, Germany). Following the transfer, membranes were then blocked using $5 \%$ low fat milk in either phosphatebuffered saline or TBST or or $3 \%$ BSA+TBST for $1 \mathrm{~h}$ at room temperature. They were subsequently incubated overnight with various primary antibodies diluted in $3 \%$ blocking buffer, $3 \%$ BSA+TBST or $1 \%$ BSA+TBST. Horseradish peroxidase coupled secondary antibodies followed by chemiluminescence (Amersham Biosciences, Millipore, Freiburg, Germany) were used to detect the antigen antibody complexes. Quantification of the obtained western blots was performed by densitometry on ImageJ.

The following antibodies were used in this study: Anti-Cdc42 mouse monoclonal (BD Transduction), Anti-beta Actin (HRP) (Abcam), Anti-beta-actin rabbit polyclonal (Sigma), Anti-XIAP mouse monoclonal (BD Pharmingen), Anti Flag (HRP) (Sigma), Anti-c-Myc mouse monoclonal (Santa Cruz), Anti-GST (Santa Cruz).

Protein-protein interaction assays. Recombinant XIAP, Cdc42 Wild Type, Cdc42Q61L and Cdc42T17N were expressed as GST fusion proteins in BL-21 Escherichia coli strain (Stratagene, Cedar Creek, TX, USA) according to manufacturer's instructions. The proteins were purified using standard protocols. Proteins were cleaved, if required from their GST tag by using Thrombin (1U) (GE Healthcare). Thrombin cleavage was performed using standard protocols. For the pulldown assays, GST PAK-PBD (Cytoskeleton) was added to the in vitro Ubiquitylation reaction of $\mathrm{Cdc} 42$ and the mix was incubated at $4{ }^{\circ} \mathrm{C}$ for $2 \mathrm{~h}$ on the rotator. A total of $10 \mu$ of Glutathione Sepharose Beads (GE Healthcare Life Sciences) which were first washed thrice with Binding Buffer (25 mM Tris- $\mathrm{HCl}$ pH7.2, $150 \mathrm{mM} \mathrm{NaCl}, 0.5 \% \mathrm{NP}-40$, $1 \mathrm{mM} \mathrm{MgCl}, 5 \%$ Glycerol) were then added to the reaction mix and was incubated for $1.5 \mathrm{~h}$ at $4{ }^{\circ} \mathrm{C}$ on the rotator. The beads were then washed thrice with $500 \mu \mathrm{l}$ of binding buffer and pelleted down at $2500 \mathrm{rpm}$ for $1 \mathrm{~min}$. A total of $50 \mu \mathrm{l}$ of sample buffer was added before boiling the samples at $100{ }^{\circ} \mathrm{C}$ for $5 \mathrm{~min}$. The interaction between the proteins of interest was then observed by SDS-PAGE.

$293 \mathrm{~T}$ cells were transfected with Cdc42 Wild Type plasmid and cells were harvested $72 \mathrm{~h}$ post transfection. RIPA buffer supplemented with phosphatase and protease inhibitors was used to lyse the cells. Lysates were then added on top of various GST tagged XIAP mutants purified in the laboratory and incubated for $2 \mathrm{~h}$ at 4 degrees. The beads were subsequently washed and finally lysed in Laemmli buffer. Samples were taken for Western Blot analysis and the GST tagged XIAP mutants were visualized using anti-GST antibody (Santa Cruz), whereas Cdc42 was visualized using anti-Cdc42 antibody (BD).

To check for the nucleotide dependence of binding, GST protein or GST tagged XIAP was first immobilized on Glutathione Sepharose beads and rotated at $4^{\circ}$ for $1 \mathrm{~h}$. One microgram of GST tagged XIAP was used for this experiment per sample. The samples were then blocked using 1\%BSA in Binding Buffer $(25 \mathrm{mM}$ Tris- $\mathrm{HCl} p \mathrm{pH} 7.2$, $150 \mathrm{mM} \mathrm{NaCl}, 0.5 \% \mathrm{NP}-40,1 \mathrm{mM} \mathrm{MgCl}, 5 \%$ Glycerol). After $1 \mathrm{~h}$ of rotation at $4^{\circ}$, the samples were washed thrice with Binding Buffer and cleaved protein was added on the beads. In all cases, either $1 \mu \mathrm{g}$ or $2 \mu \mathrm{g}$ of protein was used. Samples were rotated for $1.5 \mathrm{~h}$ at 4 degrees and then washed three times with binding buffer. In this step, $250 \mathrm{mM} \mathrm{NaCl}$ is used to minimize background binding. Laemmli buffer was added and samples were heated at $100{ }^{\circ} \mathrm{C}$ for $5 \mathrm{~min}$. The interaction between the proteins of interest was then observed by SDS-PAGE.

HIS-TUBE pulldown. HeLa cells were transfected in a 24-well plate with XIAP siRNA using Lipofectamine RNAiMAX as per manufacturer's instruction. Cells were 
transferred to $100 \mathrm{~mm}$ tissue culture plates $24 \mathrm{~h}$ later. $48 \mathrm{~h}$ post transfection, cells were treated with MG132 $(10 \mu \mathrm{M})$ for $6 \mathrm{~h}$. Then cells were lysed in RIPA buffer $(250 \mathrm{mM}$ $\mathrm{NaCl}, 50 \mathrm{mM}$ Tris- $\mathrm{HCl}, 10 \%$ Glycerin, $1 \%$ Triton X-100, pH7.5), supplemented with EDTA free Protease Cocktail inhibitor (Roche, 64693159001), and $\mathrm{N}$-ethyl maleimide $(5 \mathrm{mM})$. Equal amounts of pre-cleared lysates were incubated with $100 \mu \mathrm{g} / \mathrm{m}$ His6-TUBE1 (LifeSensors, UM201) for $15 \mathrm{~min}$ on ice. After collecting total cell lysates, the remaining lysate were incubated in Ni-NTA beads (Qiagen, Ni-NTA Superflow, 30410) and rotated overnight (cold room). Samples were washed three times with RIPA buffer containing $50 \mathrm{mM}$ imidazole and eluted in SDS buffer containing laemml and analyzed by SDS-PAGE and immunoblotted for Cdc42 using anti-Cdc42 antibody.

Mass spectrometry. Samples were analyzed using Mass Spectrometric approaches as described in ref. 4.

RT-PCR. Total RNA was extracted from cells using Trizol (Ambion/Thermo Fisher, Schwerte, Germany) or an RNA isolation kit (Thermo Fisher). In brief, cells were resuspended in Trizol, Chloroform was then added to the cell suspension and cells were vortexed for $15 \mathrm{~s}$. They were then spun down at $14000 \mathrm{rpm}$ for $15 \mathrm{~min}$ at $4{ }^{\circ}$ C. The aqueous upper phase was then transferred to sterile tubes containing Isopropanol. After incubating this mix at RT for $10 \mathrm{~min}$, the samples were again spun down at $14000 \mathrm{rpm}$ for $15 \mathrm{~min}$ at $4{ }^{\circ} \mathrm{C}$. They were then washed with $75 \%$ Ethanol and spun down at $10000 \mathrm{rpm}$ for $5 \mathrm{~min}$ at $4{ }^{\circ} \mathrm{C}$. The pellet is air dried and resuspended in $50 \mu \mathrm{l}$ of RNase free $\mathrm{dH}_{2} \mathrm{O}$. The amount of RNA was subsequently measured via NanoDrop (Thermo Fisher). When using the RNA isolation kit, standard protocol was followed. CDNA was synthesized using the RevertAid RT kit (Thermo Scientific) and manufacturer's instructions were followed. For PCR amplification, the following primers were used: Cdc42-For-1 - AGTGTTCTGC ACTTACACAGAAAG, Cdc42-Rev-1 - CTGCGGCTCTTCTTCGGT, Cdc42-For-2 AGGCTGTCAAGTATGTGGAGTG, Cdc42-Rev-2 - GGCTCTTCTTCGGTTCTGG, Cdc42-For-3 - CATCGGAATATGTACCGACTGTT, Cdc42-Rev-3 - TGCAGTATCA AAAAGTCCAAGAGTA, GAPDH-For-1 - TGCACCACCAACTGCTTAGC, GAPDHRev-1 - GGCATGGACTGTGGTCATGAG, RPS13-For-1 - CGAAAGATCTTGAG AGGAACA, RPS13-Rev-1- TCGAGCCAAACGGTGAATC

The qPCR machine was programmed as follows: 1 cycle at $50^{\circ} \mathrm{C}-2 \mathrm{~min}, 50$ cycles at $95^{\circ} \mathrm{C}-15 \mathrm{~min}, 95^{\circ} \mathrm{C}-15 \mathrm{~s}, 57^{\circ} \mathrm{C}-15 \mathrm{~s}, 72{ }^{\circ} \mathrm{C}-15 \mathrm{~s}$ and finally 1 cycle at $95^{\circ} \mathrm{C}-1 \mathrm{~min}$.

Lung colonization experiments. HeLa cells were transfected with the desired siRNAs. Forty-eight hours post transfection, cells were detached from culture plates, and $10^{6}$ cells were suspended in $100 \mu$ l of PBS before injection into the tail veins of NOD-SCID mice. After 5 weeks, lungs were fixed in 4\% PFA and analyzed for the presence of surface metastatic foci. Three independent pairs of eyes counted the foci to ensure impartiality of counts. Lungs were then hematoxylin and eosin stained and scanned.

Statistical analysis. Where applicable, data is expressed, as mean \pm S.E.M. Statistical significance was determined using an unpaired Student's $t$-test. In the event where one value was unchanged (as in Figure 1g), a paired $t$-test was used to determine significance.

\section{Conflict of Interest}

The authors declare no conflict of interest.

Acknowledgements. We would like to thank Alexandra Dimitrijevic for excellent technical assistance and Ms. Claudia Braun for her assistance with the IHC analysis of lung tumor nodules. Dr. Melzer for her help and advise with the animal experiments. We thank Professors John Silke and David Vaux for sharing XIAP knockout MEFs and CDNAs. This work is supported by a BIS-PLUS3 fellowship of the Boehringer Ingelheim Stiftuing to KR. KR is a Heisenberg Professor of the DFG (RA1739/4-1) and a GFK fellow.

1. Vega FM, Ridley AJ. Rho GTPases in cancer cell biology. FEBS Lett 2008; 582: 2093-2101.
2. Garcia-Mata R, Boulter E, Burridge K. The 'invisible hand': regulation of RHO GTPases by RHOGDIs. Nat Rev Mol Cell Biol 2011; 12: 493-504.

3. Nethe M, Hordijk PL. The role of ubiquitylation and degradation in RhoGTPase signalling. J Cell Sci 2010; 123: 4011-4018.

4. Oberoi TK, Dogan T, Hocking JC, Scholz RP, Mooz J, Anderson CL et al. IAPs regulate the plasticity of cell migration by directly targeting Rac1 for degradation. Embo J 2011; 31: 14-28.

5. Castillo-Lluva S, Tan CT, Daugaard M, Sorensen PH, Malliri A. The tumour suppressor HACE1 controls cell migration by regulating Rac1 degradation. Oncogene 2013; 32: 1735-1742.

6. Torrino S, Visvikis O, Doye A, Boyer L, Stefani C, Munro P et al. The E3 ubiquitin-ligase HACE1 catalyzes the ubiquitylation of active Rac1. Dev Cell 2011; 21: 959-965.

7. Yang L, Wang L, Zheng Y. Gene targeting of Cdc42 and Cdc42GAP affirms the critical involvement of $\mathrm{Cdc} 42$ in filopodia induction, directed migration, and proliferation in primary mouse embryonic fibroblasts. Mol Biol Cell 2006; 17: 4675-4685.

8. Garvalov BK, Flynn KC, Neukirchen D, Meyn L, Teusch N, WuX et al. Cdc42 regulates cofilin during the establishment of neuronal polarity. J Neurosci 2007; 27: 13117-13129.

9. El-Sibai M, Nalbant P, Pang H, Flinn RJ, Sarmiento C, Macaluso F et al. Cdc42 is required for EGF-stimulated protrusion and motility in MTLn3 carcinoma cells. J Cell Sci 2007; 120: 3465-3474.

10. Srinivasula SM, Ashwell JD. IAPs: what's in a name? Mol Cell 2008; 30: 123-135.

11. Vaux DL, Silke J. IAPs, RINGs and ubiquitylation. Nat Rev Mol Cell Biol 2005; 6 : 287-297.

12. Hochstrasser M. Origin and function of ubiquitin-like proteins. Nature 2009; 458: 422-429.

13. Varshavsky A. The early history of the ubiquitin field. Protein Sci 2006; 15: 647-654.

14. Haglund K, Dikic I. Ubiquitylation and cell signaling. Embo J 2005; 24: 3353-3359.

15. Gyrd-Hansen M, Meier P. IAPs: from caspase inhibitors to modulators of NF-kappaB, inflammation and cancer. Nat Rev Cancer 2010; 10: 561-574.

16. Dogan T, Harms GS, Hekman M, Karreman C, Oberoi TK, Alnemri ES et al. X-linked and cellular IAPs modulate the stability of C-RAF kinase and cell motility. Nat Cell Biol 2008; 10: 1447-1455.

17. Takeda AN, Oberoi-Khanuja TK, Glatz G, Schulenburg K, Scholz RP, Carpy A et al. Ubiquitin-dependent regulation of MEKK2/3-MEK5-ERK5 signaling module by XIAP and CIAP1. Embo J 2014; 33: 1784-1801.

18. Doye A, Boyer L, Mettouchi A, Lemichez E. Ubiquitin-mediated proteasomal degradation of Rho proteins by the CNF1 toxin. Methods Enzymol 2006; 406: 447-456.

19. Reymond N, Im JH, Garg R, Vega FM, Borda d'Agua B, Riou P et al. Cdc42 promotes transendothelial migration of cancer cells through beta1 integrin. J Cell Biol 2012; 199: 653-668.

20. Wei J, Mialki RK, Dong S, Khoo A, Mallampalli RK, Zhao Y et al. A new mechanism of RhoA ubiquitination and degradation: roles of SCF(FBXL19) E3 ligase and Erk2. Biochim Biophys Acta 2013; 1833: 2757-2764

21. Wang HR, Ogunjimi AA, Zhang Y, Ozdamar B, Bose R, Wrana JL. Degradation of RhoA by Smurf1 ubiquitin ligase. Methods Enzymol 2006; 406: 437-447.

22. Zhao J, Mialki RK, Wei J, Coon TA, Zou C, Chen BB et al. SCF E3 ligase F-box protein complex SCF(FBXL19) regulates cell migration by mediating Rac1 ubiquitination and degradation. FASEB J 2013; 27: 2611-2619.

23. Castillo-Lluva S, Tatham MH, Jones RC, Jaffray EG, Edmondson RD, Hay RT et al. SUMOylation of the GTPase Rac1 is required for optimal cell migration. Nat Cell Biol 2010; 12: $1078-1085$

24. Liu J, Zhang D, Luo W, Yu Y, Yu J, Li J et al. X-linked inhibitor of apoptosis protein (XIAP) mediates cancer cell motility via Rho GDP dissociation inhibitor (RhoGDI)-dependent regulation of the cytoskeleton. J Biol Chem 2011; 286: 15630-15640.

25. Marivin A, Berthelet J, Cartier J, Paul C, Gemble S, Morizot A et al. clAP1 regulates TNF-mediated cdc42 activation and filopodia formation. Oncogene 2014; 33: 5534-5545.

(i) Cell Death and Disease is an open-access journal published by Nature Publishing Group. This work is licensed under a Creative Commons Attribution 4.0 International License. The images or other third party material in this article are included in the article's Creative Commons license, unless indicated otherwise in the credit line; if the material is not included under the Creative Commons license, users will need to obtain permission from the license holder to reproduce the material. To view a copy of this license, visit http://creativecommons.org/licenses/by/4.0/

(C) The Author(s) 2017

Supplementary Information accompanies this paper on Cell Death and Disease website (http://www.nature.com/cddis) 